\title{
Mitteilungsseiten DGINA
}

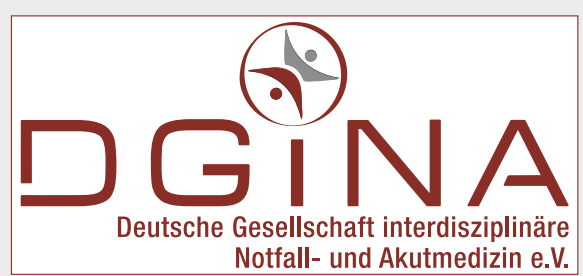

Notfall Rettungsmed 2020 $23: 313-314$

https://doi.org/10.1007/s10049-020-00712-9

(c) Springer Medizin Verlag GmbH, ein Teil von Springer Nature 2020
Deutsche Gesellschaft interdisziplinäre Notfall- und

Akutmedizin (DGINA) e.V.

Geschäftsstelle

Hohenzollerndamm 152 14199 Berlin

\section{Redaktion:}

Martin Pin (V.i. S.d.P.)

Florence-Nightingale-Krankenhaus

Kaiserswerther Diakonie

Kreuzbergstraße 79, 40489 Düsseldorf

Presse- und Öffentlichkeitsarbeit: Dr. Caroline Mayer

E-Mail: presse@dgina.de

\section{Klinikpersonal als potenzielle Second Victims der COVID-19- Pandemie - was ist zu tun?}

\begin{abstract}
Wie können Ärztinnen, Ärzte und Pflegekräfte in Zeiten der COVID19-Pandemie ihre Resilienz stärken? Und wie kann Helfern selbst geholfen werden, die in dieser Situation Belastungsreaktionen zeigen? Dazu haben wir Prof. Dr. Reinhard Strametz befragt. Er hat für das Aktionsbündnis Patientensicherheit (APS e.V.) eine entsprechende Handlungsempfehlung mitverfasst.
\end{abstract}

Herr Professor Strametz, Sie sagen, dass viele Behandler in der Corona-Pandemie zu Second Victims werden. Was bedeutet das?

Strametz: Ursprünglich ist der Begriff „Second Victim“ für Behandelnde geprägt worden, denen bei ihrer Arbeit ein Fehler unterlaufen ist und die sich deswegen schuldig fühlen. Seit über zehn Jahren verwenden wir die Bezeichnung aber auch für Helfende aller Berufsgruppen, die in außergewöhnlichen klinischen Situationen - wie wir sie jetzt im Rahmen der Pandemie erleben stark emotional belastet werden. Das kann die Betroffenen unter Umständen massiv schädigen.

\section{Inwiefern?}

Strametz: Die Folgen reichen von einfachen Belastungsreaktionen, die in vielen Fällen zum

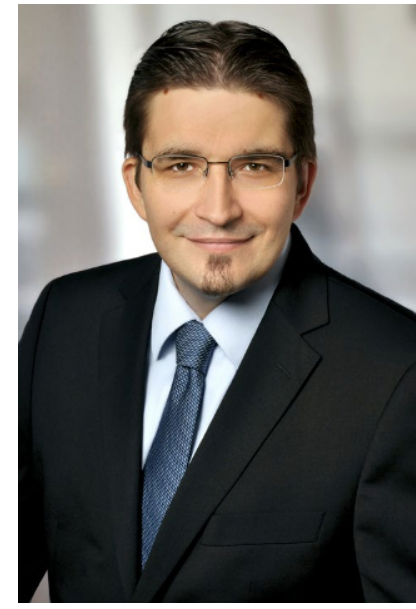

A Prof. Dr. Reinhard Strametz hat die Professur „Medizin für Ökonomen" an der Hochschule RheinMain. Er ist Beisitzer im Vorstand des Aktionsbündnisses Patientensicherheit e.V.

Glück vorübergehender Natur sind, bis hin zu einer chronischen Reaktion im psychosomatischen Bereich. Es können Schlafstörungen und Flashbacks auftreten, manche Betroffene flüchten sich in Ausweichstrategien und beginnen, Alkohol oder Drogen zu konsumieren. In einigen Fällen führt die akute Gesundheitsbelastung zur Berufsaufgabe oder sogar zum Suizid. Aus Italien und New York haben wir solche erschütternden Meldungen während der Pandemie ja leider schon gehört.
Kann man bereits abschätzen, wie groß das Ausmaß des Problems in der aktuellen COVID19-Pandemie ist?

Strametz: Dazu haben wir noch keine belastbaren Zahlen. Aber wir wissen aus vergleichbaren Ereignissen wie beispielsweise dem 11. September oder dem ICE-Unglück von Eschede, dass etwa $50 \%$ aller Helfenden - unabhängig von der Berufsgruppe akut belastet werden bis hin zur posttraumatischen Belastungsstörung. Die jetzige Situation des Massenanfalls Infizierter ist natürlich noch einmal besonders. Ein Massenanfall von Verletzten ist vergleichsweise schnell abgearbeitet. Bei einer Pandemie kommen aber immer mehr und mehr Patienten. Das ist eine chronische Belastung, die zusätzlich noch mit der Angst vor der Überforderung des Gesundheitswesens einhergeht. Insofern kann man davon ausgehen, dass die Zahl der Betroffenen jetzt sogar höher ist und dass wir noch lange mit den Folgen zu kämpfen haben werden.

Was raten Sie Behandlern, die sich jetzt überlastet fühlen?

Strametz: Ich muss mir als Erstes klarmachen, dass es eine völlig normale menschliche Reaktion ist und kein Zeichen von Schwäche oder Inkompetenz. Eine der größten Hilfestellungen ist das Gespräch mit Kolleginnen und Kollegen. In einigen Kliniken sind solche Gespräche nach belastenden Ereignissen schon etabliert. Wo das noch nicht der Fall ist, empfehle ich zum Beispiel das Projekt „EMPTY“, den „Emergency Medicine Problem Talk der YoungDGINA“. Das ist ein niedrigschwelliges Gesprächsangebot insbesondere für junge Helfende - von Peer zu Peer. Wenn es vor Ort bereits Kriseninterventionsteams in Kooperation mit dem Rettungsdienst gibt, können auch diese Teams helfen, selbstverständlich auch die Krankenhausseelsorge. Darüber hinaus ist Selbstfürsorge sehr wichtig: sich den Freiraum nehmen, sich selbst etwas Gutes zu tun. Nur gesunde Helfende sind auch gute Helfende. Für sich selbst Sorge zu tragen, gerade in solch einer Krise, ist eine wesentliche Voraussetzung dafür, in kritischen Situationen auch gut für Patientinnen und Patienten sorgen zu können.

Haben Sie konkrete Tipps, wie man gut für sich selber sorgen und die eigene Resilienz stärken kann?

Strametz: Jeder muss natürlich seinen eigenen Weg finden und lernen, was ihm oder ihr guttut. Nichtsdestotrotz gibt es auch dafür entsprechende Hilfsangebote. Ich kann die aus meiner Sicht fachlich ausgezeichneten Informationsblätter des Vereins PSU Akut e. V. empfehlen. Zum Beispiel „Möglichkeiten der Stressreduktion für medizinisches Personal in Zeiten von COVID-19“. Die Stärkung der Resilienz ist aber nicht nur eine Sache des Einzelnen. Dazu können und müssen auch Führungs- 


\section{Weiterführende Informationen}

Aktionsbündnis Patientensicherheit: www.aps-ev.de -> Aktuelles -> \#Corona

EMPTY: www.youngdgina.com

PSU-AKUT: www.psu-akut.de

DGINA Notfallcampus: Aktuelle Informationen zu COVID-19 Während der COVID-19-Pandemie hat der Notfallcampus sein Präsenzseminarprogramm eingestellt. Trotzdem stellt die Institution weiterhin aktuelles Wissen und Fortbildungsinhalte zur Notfallmedizin zur Verfügung: Auf der Website www.notfall-campus.de sind aktuelle Informationen zu COVID-19 zu finden, die laufend ergänzt werden. So können Interessierte dort unter anderem alle gemeinsamen COVID19-Webinare von NOW TO GO und der DGINA ansehen. Die Videos werden ergänzt durch Download-Material, Zahlen, Daten und Fakten, Links zu medizinischen Übersichtsseiten, Lehrvideos und Podcasts. Themen sind unter anderem die Intubation unter COVID-19, Ultraschalldiagnostik bei COVID-19 oder Hygiene in der ZNA.

kräfte einiges beitragen, zum Beispiel durch eine gute Fehlerkultur und eine gute Krisenkommunikation, wie wir in unserer Handlungsempfehlung des APS aufzeigen.

Fällt es vielen Helferinnen und Helfern heute immer noch schwer, eigene Schwächen zu akzeptieren und Gesprächsangebote wahrzunehmen?

Strametz: Gerade Ärztinnen und Ärzte sind leider häufig die schlechtesten Patienten, weil sie darauf trainiert werden, dass sie selbst die Gesunden und die Patienten die Kranken sind. Wer Hemmungen hat, sich helfen zu lassen, dem kann ich nur sagen: Belastungsreaktionen sind völlig normal, bis zu $50 \%$ unserer Kolleginnen und Kollegen erleben das gerade und müssen das irgendwie verarbeiten. Das gehört dazu und es sollte eine Selbstverständlichkeit sein, darüber $\mathrm{zu}$ sprechen. Falscher Stolz schadet nur. Denn wer sich dem Thema stellt, kann auch gestärkt aus einem solchen Erlebnis herausgehen. Ich sehe in der aktuellen Situation somit auch eine Chance: Da jetzt alle gemeinsam diese Erfahrungen machen, kann sich in Zukunft Vieles verbessern, auch in der Kommunikation über dieses sensible Thema.

Mit Prof. Dr. Reinhard Strametz sprach Dr. Caroline Mayer 